J. Korean Math. Soc. 51 (2014), No. 6, pp. 1209-1220

http://dx.doi.org/10.4134/JKMS.2014.51.6.1209

\title{
GLOBAL GRADIENT ESTIMATES FOR NONLINEAR ELLIPTIC EQUATIONS
}

\author{
SEunguin Ryu
}

\begin{abstract}
We prove global gradient estimates in weighted Orlicz spaces for weak solutions of nonlinear elliptic equations in divergence form over a bounded non-smooth domain as a generalization of Calderón-Zygmund theory. For each point and each small scale, the main assumptions are that nonlinearity is assumed to have a uniformly small mean oscillation and that the boundary of the domain is sufficiently flat.
\end{abstract}

\section{Introduction}

Let $\Omega$ be a bounded domain in $\mathbb{R}^{n}, n \geq 2$, with its non-smooth boundary $\partial \Omega$ and let $F=F(x): \Omega \rightarrow \mathbb{R}^{n}$ be a given vector-valued function at least in $L^{2}\left(\Omega, \mathbb{R}^{n}\right)$. With these notations, consider the following nonlinear elliptic equation in divergence form:

$$
\left\{\begin{array}{rlrl}
\operatorname{div} \mathbf{a}(D u, x) & =\operatorname{div} F & \text { in } \quad \Omega, \\
u & =0 & \text { on } & \partial \Omega,
\end{array}\right.
$$

where the nonlinearity $\mathbf{a}=\mathbf{a}(\xi, x): \mathbb{R}^{n} \times \mathbb{R}^{n} \rightarrow \mathbb{R}^{n}$ is measurable in $x$ for all $\xi \in \mathbb{R}^{n}$ and continuous in $\xi$ for almost all $x \in \mathbb{R}^{n}$. Here assume standard monotonicity and growth conditions on a as follows: For some positive constants $c_{0}$ and $c_{1}$,

$$
\left\{\begin{array}{l}
c_{0}|\xi-\eta|^{2} \leq[\mathbf{a}(\xi, x)-\mathbf{a}(\eta, x)] \cdot(\xi-\eta) \\
|\mathbf{a}(\xi, x)|+|\xi|\left|D_{\xi} \mathbf{a}(\xi, x)\right| \leq c_{1}|\xi|
\end{array}\right.
$$

for all $\xi, \eta \in \mathbb{R}^{n}$ and for almost every $x \in \mathbb{R}^{n}$.

As usual, we consider a weak solution $u \in H_{0}^{1}(\Omega)$, which means that the following integral formula holds:

$$
\int_{\Omega} \mathbf{a}(D u, x) \cdot D \varphi d x=\int_{\Omega} F \cdot D \varphi d x, \quad \forall \varphi \in H_{0}^{1}(\Omega) .
$$

Received February 14, 2014.

2010 Mathematics Subject Classification. 35J60, 46E30.

Key words and phrases. nonlinear elliptic equation, global gradient estimate, CalderónZygmund theory, BMO space, Reifenberg flat domain.

This work was supported by the 2013 Research Fund of the University of Seoul. 
The existence and uniqueness of a weak solution to problem (1.1) can be obtained by the Minty-Browder method for monotone operators, see [9, 20], under the assumption $F \in L^{2}\left(\Omega, \mathbb{R}^{n}\right)$, with the estimate

$$
\left\||D u|^{2}\right\|_{L^{1}\left(\Omega, \mathbb{R}^{n}\right)} \leq c\left\||F|^{2}\right\|_{L^{1}\left(\Omega, \mathbb{R}^{n}\right)},
$$

the constant $c$ is independent of $u$ and $F$.

In this paper

$$
B_{\rho}(y)=\left\{x \in \mathbb{R}^{n}:|x-y|<\rho\right\}
$$

denotes the open ball on $\mathbb{R}^{n}$ centered $y \in \mathbb{R}^{n}$ and radius $\rho>0$ and $|E|$ denotes the $n$-dimensional Lebesgue measure of a set $E \subset \mathbb{R}^{n}$. With the notation, the regularity assumption on the nonlinearity $\mathbf{a}=\mathbf{a}(\xi, x)$ and a finer geometric assumption on the domain $\Omega$ are introduced. First set

$$
\theta\left(\mathbf{a} ; B_{\rho}(y)\right)(x)=\sup _{\xi \in \mathbb{R}^{n} \backslash\{0\}} \frac{\left|\mathbf{a}(\xi, x)-\overline{\mathbf{a}}_{B_{\rho}(y)}(\xi)\right|}{|\xi|},
$$

where

$$
\overline{\mathbf{a}}_{B_{\rho}(y)}(\xi)=f_{B_{\rho}(y)} \mathbf{a}(\xi, x) d x=\frac{1}{\left|B_{\rho}(y)\right|} \int_{B_{\rho}(y)} \mathbf{a}(\xi, x) d x
$$

is the integral average of $\mathbf{a}(\xi, x)$ in the variable $x$ over $B_{\rho}(y)$ for fixed $\xi \in \mathbb{R}^{n}$. The function $\theta\left(\mathbf{a} ; B_{\rho}(y)\right)$ provides the measurement of the oscillation of $\frac{\mathbf{a}(\xi, x)}{|\xi|}$ in the variable $x$ over $B_{\rho}(y)$, uniformly in $\xi$.

Definition 1.1. A vector field $\mathbf{a}$ is said to be $(\delta, R)$-vanishing if

$$
\sup _{0<\rho \leq R} \sup _{y \in \mathbb{R}^{n}} f_{B_{\rho}(y)} \theta\left(\mathbf{a} ; B_{\rho}(y)\right)(x) d x \leq \delta .
$$

To measure the deviation of $\partial \Omega$ from being an $(n-1)$-dimensional affine space at each scale $\rho>0$, use the following so-called "Reifenberg flatness".

Definition 1.2. A bounded domain $\Omega$ is said to be $(\delta, R)$-Reifenberg flat if for every $x \in \partial \Omega$ and every $\rho \in(0, R]$, there exists a coordinate system $\left\{y_{1}, \ldots, y_{n}\right\}$, which can depend on $\rho$ and $x$ such that $x=0$ in this coordinate system and that

$$
B_{\rho}(0) \cap\left\{y_{n}>\delta \rho\right\} \subset B_{\rho}(0) \cap \Omega \subset B_{\rho}(0) \cap\left\{y_{n}>-\delta \rho\right\} .
$$

Remark 1.3. The above definition warrants a few comments. Because the main problem (1.1) has a scaling invariance property, the constant $R$ can be taken as 1 or any other constant greater than 1 . However, the constant $\delta$ is a small positive constant still invariant under such scaling (see Lemma 2.1). In fact, the Reifenberg flatness (1.3) is meaningful when $0<\delta<\frac{1}{8}$, see [25], and with such small $\delta$, these flatness conditions mean that the deviation of $\partial \Omega$ from being an $(n-1)$-dimensional affine space is small enough at each scale $\rho>0$. In addition, by (1.3), the following measure density condition is obtained:

$$
\left|\Omega \cap B_{\rho}(y)\right| \geq\left(\frac{1-\delta}{2}\right)^{n}\left|B_{\rho}(y)\right| \geq\left(\frac{7}{16}\right)^{n}\left|B_{\rho}(y)\right|
$$


for all $y \in \Omega$ and $\rho \in(0, R)$.

Here the purpose is to generalize Calderón-Zygmund-type estimates of the gradient of a weak solution of (1.1) in weighted Orlicz spaces. Because these Calderón-Zygmund-type estimates play an important role in regularity theory with Hölder estimates, studies have examined for classical $L^{p}$ estimates or their generalizations (e.g., $[8,11,13,21,26]$ ).

From a technical point of view, this paper appropriately applies the approach introduced in [10] and later developed in [3, 6, 7]. Although the main tools are the Hardy-Littlewood maximal function and the Calderón-ZygmundKrylov-Safonov-type decomposition, the general theory of singular integrals employed in $[15,22,24]$ is not used. Note that this approach is useful even when considering global estimates with non-smooth boundaries. It should be point out that one may obtain the same results based on the so-called "Harmonicanalysis-free" technique in [1]. This approach is quite effective for $L^{p}$ regularity estimates for nonlinear parabolic problems with no invariance property under normalization, see $[2,4,14]$. In addition, note that the so-called "sharp maximal function method", first introduced in [18] and later modified in $[11,12,19]$, is useful when differential operators related to problems are bounded and linear.

The Muchenhoupt weight is now introduced. A positive and locally integrable function $w$ on $\mathbb{R}^{n}$ is said to be of class $A_{p}, 1<p<\infty$, if

$$
\sup \left(\frac{1}{|B|} \int_{B} w(x) d x\right)\left(\frac{1}{|B|} \int_{B} w(x)^{\frac{-1}{p-1}} d x\right)^{p-1} \leq A<\infty,
$$

where the supremum runs over all balls $B$ formed by $B=B_{\rho}(y)$. Note that the smallest constant $A$ for which (1.4) holds is denoted by $[w]_{p}$. Given $w \in A_{p}$ and a measurable set $E \subset \mathbb{R}^{n}$, we use the notation

$$
w(E)=\int_{E} w(x) d x
$$

to denote the $w$-measure of the set $E$. On the other hand, there is another way to define the $A_{p}$ class: That is, the weight $w$ belongs to $A_{p}$ if and only if

$$
\left(\frac{1}{|B|} \int_{B} f(x) d x\right)^{p} \leq \frac{c}{w(B)} \int_{B}(f(x))^{p} w(x) d x
$$

holds for all positive functions $f$ and all balls $B$. The smallest constant $c$ for which (1.5) is valid equals $[w]_{p}$. As a direct consequence of (1.5), the $A_{p}$ weight has a doubling property (see the first inequality $(*)$ of $(1.6)$ ). First, a remarkable feature of $A_{p}$ weights is that they have the reverse Hölder property. That is, for $w \in A_{p}(1<p<\infty)$, there exists a small positive constant $\epsilon_{0}$ depending only on $n, p$ and $[w]_{p}$ such that $w \in A_{p-\epsilon_{0}}$ with the estimate $[w]_{p-\epsilon_{0}}<c[w]_{p}$ for some $c=c\left(n, p,[w]_{p}\right)>0$. The unification of the doubling and reverse Hölder properties produces the following comparability between 
the $w$-measure and the Lebesgue measure:

$$
\frac{1}{[w]_{p}}\left(\frac{|E|}{|B|}\right)^{p} \stackrel{(*)}{\leq} \frac{w(E)}{w(B)} \leq c_{2}\left(\frac{|E|}{|B|}\right)^{\tau_{1}}, \quad E \subset B,
$$

for some constants $c_{2}>1$ and $\tau_{1} \in(0,1)$. We remark that these $c_{2}$ and $\tau_{1}$ depend only on $n, p$, and $[w]_{p}$ and thus not on $E$ and $B$.

We now turn to Orlicz spaces. The function $\Phi:[0, \infty) \rightarrow[0, \infty)$ is said to be a Young function if $\Phi$ is increasing, convex, and satisfies

$$
\Phi(0)=0, \Phi(\infty)=\lim _{\rho \rightarrow+\infty} \Phi(\rho)=+\infty, \lim _{\rho \rightarrow 0+} \frac{\Phi(\rho)}{\rho}=0, \lim _{\rho \rightarrow+\infty} \frac{\Phi(\rho)}{\rho}=+\infty .
$$

Throughout the paper, the Young function $\Phi$ is assumed to satisfy $\Delta_{2}$ and $\nabla_{2}$ conditions, denoted by $\Phi \in \Delta_{2} \cap \nabla_{2}$,

- $\left(\Delta_{2}\right.$-condition) there exists $\mu>1$ such that $\Phi(2 \rho) \leq \mu \Phi(\rho)$ for all $\rho>0$;

- $\left(\nabla_{2}\right.$-condition $)$ there exists $\nu>1$ such that $2 \nu \Phi(\rho) \leq \Phi(\nu \rho)$ for all $\rho>0$.

We next define the lower index of $\Phi$, denoted by $i(\Phi)$, by

$$
i(\Phi)=\lim _{\lambda \rightarrow 0+} \frac{\log \left(h_{\Phi}(\lambda)\right)}{\log \lambda}=\sup _{0<\lambda<1} \frac{\log \left(h_{\Phi}(\lambda)\right)}{\log \lambda},
$$

where

$$
h_{\Phi}(\lambda)=\sup _{\rho>0} \frac{\Phi(\lambda \rho)}{\Phi(\rho)} \quad(\lambda>0) .
$$

For example, $i(\Phi)=q$ if $\Phi(\rho)=\rho^{q}$ with $q>1$. In addition, this $\Delta_{2} \cap \nabla_{2^{-}}$ condition ensures that the Young function increases moderately. That is, there are two constants $q_{0}$ and $q_{1}$ with $1<q_{0} \leq q_{1}<\infty$ such that

$$
\frac{1}{c_{3}} \min \left\{\lambda^{q_{0}}, \lambda^{q_{1}}\right\} \Phi(\rho) \leq \Phi(\lambda \rho) \leq c_{3} \max \left\{\lambda^{q_{0}}, \lambda^{q_{1}}\right\} \Phi(\rho), \quad \lambda, \rho \geq 0,
$$

where the constant $c_{3}$ is independent of $\lambda$ and $\rho$. In fact, the index number $i(\Phi)$ is equal to the supremum of $q_{0}$ satisfying (1.7). Finally, we would like to mention that the $\Delta_{2} \cap \nabla_{2}$-condition is unavoidable for the type of regularity considered here, see [27].

The condition $w \in A_{i(\Phi)}$ is the main assumption on the Muckenhoupt weight $w(x)$. Because $\Phi \in \Delta_{2} \cap \nabla_{2}, 1<i(\Phi)<\infty$. It is worth summarizing an important property of the $A_{i(\Phi)}$ weight $w$ considered here. There exists a small positive constant $\epsilon_{0}$ depending the index $i(\Phi)$ and the dimension such that $w \in A_{i(\Phi)-\epsilon_{0}}$ with the estimate $[w]_{i(\Phi)-\epsilon_{0}} \leq c_{n, i(\Phi)}[w]_{i(\Phi)}$. Consequently,

$$
[w]_{i(\Phi)} \leq[w]_{i(\Phi)-\epsilon_{0}} \leq c[w]_{i(\Phi)}
$$

and therefore assume that

$$
\lambda^{i(\Phi)-\epsilon_{0}} \Phi(t) \leq c \Phi(\lambda t), \quad \lambda \geq 1, t \geq 0 .
$$

We refer to $[16,17]$ for a more in-depth discussion on the condition $w \in A_{i(\Phi)}$. 
We now ready to introduce the weighted Orlicz space considered here. For a Young function $\Phi \in \Delta \cap \nabla_{2}$ and a positive and locally integrable function $w=$ $w(x) \in A_{i(\Phi)}$, the weighted Orlicz space $L_{w}^{\Phi}(\Omega)$ is the class of all measurable functions $g: \Omega \rightarrow \mathbb{R}$ satisfying

$$
\int_{\Omega} \Phi(|g(x)|) w(x) d x<+\infty .
$$

Indeed, the weighted Luxemburg norm,

$$
\|g\|_{L_{w}^{\Phi}(\Omega)}=\inf \left\{\kappa>0: \int_{\Omega} \Phi\left(\frac{|g(x)|}{\kappa}\right) w(x) d x \leq 1\right\},
$$

is well-defined as a norm, up to equal almost everywhere, on $L_{w}^{\Phi}(\Omega)$, see [17].

By the convexity of $\Phi$ and the estimate (1.7), the following is obtained:

$$
\begin{aligned}
& \frac{1}{c_{3}} \min \left\{\|g\|_{L_{w}^{\Phi}(\Omega)}^{q_{0}},\|g\|_{L_{w}^{\Phi}(\Omega)}^{q_{1}}\right\} \\
\leq & \int_{\Omega} \Phi(|g(x)|) w(x) d x \leq c_{3} \max \left\{\|g\|_{L_{w}^{\Phi}(\Omega)}^{q_{0}},\|g\|_{L_{w}^{\Phi}(\Omega)}^{q_{1}}\right\} .
\end{aligned}
$$

If $\|g\|_{L_{w}^{\Phi}(\Omega)} \leq 1$, then it follows from (1.7) that

$$
\frac{1}{c_{3}}\left(\frac{1}{\|g\|_{L_{w}^{\Phi}(\Omega)}+\epsilon}\right)^{q_{0}} \int_{\Omega} \Phi(|g(x)|) w(x) d x \leq \int_{\Omega} \Phi\left(\frac{|g(x)|}{\|g\|_{L_{w}^{\Phi}(\Omega)}+\epsilon}\right) w(x) d x \leq 1
$$

and that

$$
1<\int_{\Omega} \Phi\left(\frac{|g(x)|}{\|g\|_{L_{w}^{\Phi}(\Omega)}-\epsilon}\right) w(x) d x \leq c_{3}\left(\frac{1}{\|g\|_{L_{w}^{\Phi}(\Omega)}-\epsilon}\right)^{q_{1}} \int_{\Omega} \Phi(|g(x)|) w(x) d x
$$

for all sufficiently small $\epsilon>0$. Therefore,

$$
\frac{1}{c_{3}}\|g\|_{L_{w}^{\Phi}(\Omega)}^{q_{1}} \leq \int_{\Omega} \Phi(|g(x)|) w(x) d x \leq c_{3}\|g\|_{L_{w}^{\Phi}(\Omega)}^{q_{0}}
$$

provided that $\|g\|_{L_{w}^{\Phi}(\Omega)} \leq 1$. Similarly, we have

$$
\frac{1}{c_{3}}\|g\|_{L_{w}^{\Phi}(\Omega)}^{q_{0}} \leq \int_{\Omega} \Phi(|g(x)|) w(x) d x \leq c_{3}\|g\|_{L_{w}^{\Phi}(\Omega)}^{q_{1}}
$$

in the case $\|g\|_{L_{w}^{\Phi}(\Omega)}>1$. This finishes the proof for (1.9).

We are now ready to state the main result.

Theorem 1.4. Given a Young function $\Phi \in \Delta_{2} \cap \nabla_{2}$, let $w \in A_{i(\Phi)}$. Suppose that $|F|^{2} \in L_{w}^{\Phi}(\Omega)$ and $u \in H_{0}^{1}(\Omega)$ is a weak solution of (1.1). Then there exists a small positive constant $\delta=\delta\left(c_{0}, c_{1}, n, \Phi, w\right)$ such that if $\mathbf{a}$ is $(\delta, R)$-vanishing and $\Omega$ is $(\delta, R)$-Reifenberg flat, then $|D u|^{2} \in L_{w}^{\Phi}(\Omega)$ with the estimate

$$
\left\||D u|^{2}\right\|_{L_{w}^{\Phi}(\Omega)} \leq c\left\||F|^{2}\right\|_{L_{w}^{\Phi}(\Omega)},
$$

the constant $c$ depending on $c_{0}, c_{1}, n, R, \Phi, w$, and $\Omega$. 
We remark that the present result is a natural extension of previous research $[9,20]$. In fact, the problem (1.1) under the same conditions is considered in unweighted [9] and weighted [20] Lebesgue spaces.

Before ending this section, we check the existence and uniqueness of a weak solution. The following lemma ensures that for each $F(x)$ with $|F|^{2} \in L_{w}^{\Phi}(\Omega)$, the problem (1.1) has a unique weak solution.

Lemma 1.5. Let $\Phi \in \Delta_{2} \cap \nabla_{2}$ and $w \in A_{i(\Phi)}$. If $|F|^{2} \in L_{w}^{\Phi}(\Omega)$, then $|F|^{2} \in$ $L^{1}(\Omega)$, and

$$
\int_{\Omega}|F(x)|^{2} d x \leq c\left[\left(\int_{\Omega} \Phi\left(|F|^{2}\right) w(x) d x\right)^{\frac{1}{q_{0}}}+\left(\int_{\Omega} \Phi\left(|F|^{2}\right) w(x) d x\right)^{\frac{1}{q_{1}}}\right],
$$

where $q_{0}$ and $q_{1}$ are defined in (1.7).

Proof. We first recall the self-improving property of $A_{i(\Phi)}$-weight, that is, $w \in$ $A_{i(\Phi)-\epsilon_{0}}$ with (1.8). Set $f(x)=|F(x)|^{2}$. With a direct calculation,

$$
\begin{aligned}
\int_{\{\Omega:|f| \geq 1\}}|f(x)| d x & =\int_{\{\Omega:|f| \geq 1\}}|f(x)| w(x)^{\frac{1}{i(\Phi)-\epsilon_{0}}} w(x)^{-\frac{1}{i(\Phi)-\epsilon_{0}}} d x \\
& \leq\left(\int_{\{\Omega:|f| \geq 1\}}|f(x)|^{i(\Phi)-\epsilon_{0}} w(x) d x\right)^{\frac{1}{i(\Phi)-\epsilon_{0}}} \\
& \times\left(\int_{\Omega} w(x)^{\frac{-1}{i(\Phi)-\epsilon_{0}-1}} d x\right)^{\frac{i(\Phi)-\epsilon_{0}-1}{i(\Phi)-\epsilon_{0}}} \\
& \leq \frac{|\Omega|[w]_{i(\Phi)-\epsilon_{0}}}{w(\Omega)^{\frac{1}{i(\Phi)-\epsilon_{0}}}}\left(\int_{\{\Omega:|f| \geq 1\}}|f(x)|^{i(\Phi)-\epsilon_{0}} w(x) d x\right)^{\frac{1}{i(\Phi)-\epsilon_{0}}} .
\end{aligned}
$$

It follows from (1.8) that

$$
|f(x)|^{i(\Phi)-\epsilon_{0}} \leq \frac{c}{\Phi(1)} \Phi(|f(x)|) \quad \text { if } \quad|f(x)| \geq 1
$$

and so

$$
\int_{\{\Omega:|f| \geq 1\}}|f(x)| d x \leq c\left(\int_{\Omega} \Phi(|f(x)|) w(x) d x\right)^{\frac{1}{i(\Phi)-\epsilon_{0}}} .
$$

On the other hand, it follows from $i(\Phi) \leq q_{1}$ that $w \in A_{q_{1}}$ with $[w]_{q_{1}} \leq[w]_{i(\Phi)}$. Because, by (1.7),

$$
\begin{gathered}
|f(x)|^{q_{1}} \leq \frac{c}{\Phi(1)} \Phi(|f(x)|) \quad \text { if } \quad|f(x)| \leq 1, \\
\int_{\{\Omega:|f| \leq 1\}}|f(x)| d x \leq c\left(\int_{\Omega} \Phi(|f(x)|) w(x) d x\right)^{\frac{1}{q_{1}}} .
\end{gathered}
$$


Since $\epsilon_{0}$ is small enough, we get

$$
\int_{\Omega}|f(x)| d x \leq c\left[\left(\int_{\Omega} \Phi(|f(x)|) w(x) d x\right)^{\frac{1}{q_{0}}}+\left(\int_{\Omega} \Phi(|f(x)|) w(x) d x\right)^{\frac{1}{q_{1}}}\right] .
$$

\section{Preliminary tools}

We begin this section with the following invariance property under normalization and scaling. The proof follows by direct computations (for further details, see [9]).

Lemma 2.1. Let $u$ be a weak solution to the problem (1.1). Assume that the nonlinearity $\mathbf{a}(\xi, x)$ satisfies (1.2) and is $(\delta, R)$-vanishing. For each $\lambda>1$ and $0<r<1$, define the rescaled maps

$$
\tilde{\mathbf{a}}(\xi, x)=\frac{\mathbf{a}(\lambda \xi, r x)}{\lambda}, \quad \tilde{\Omega}=\left\{\frac{1}{r} x: x \in \Omega\right\}, \quad \tilde{u}(x)=\frac{u(r x)}{\lambda r}, \quad \tilde{F}(x)=\frac{F(r x)}{\lambda} .
$$

Then

(1) $\tilde{u} \in H_{0}^{1}(\tilde{\Omega})$ is the weak solution of

$$
\operatorname{div} \tilde{\mathbf{a}}(D \tilde{u}, x)=\operatorname{div} \tilde{F} \quad \text { in } \tilde{\Omega},
$$

(2) $\tilde{\mathbf{a}}(\xi, x)$ satisfies the structural assumption (1.2) with the same constants $c_{0}$ and $c_{1}$,

(3) $\tilde{\mathbf{a}}$ is $\left(\delta, \frac{R}{r}\right)$-vanishing and $\tilde{\Omega}$ is $\left(\delta, \frac{R}{r}\right)$-Reifenberg flat.

We now recall the Hardy-Littlewood maximal function and its basic properties. Let $g$ be a locally integrable function on $\mathbb{R}^{n}$. Then the Hardy-Littlewood maximal function is given by

$$
(\mathcal{M g})(x)=\sup _{\rho>0} f_{B_{\rho}(x)}|g(y)| d y=\sup _{\rho>0} \frac{1}{\left|B_{\rho}(x)\right|} \int_{B_{\rho}(x)}|g(y)| d y .
$$

If $g$ is defined only on a bounded subset of $\mathbb{R}^{n}$, then we define as

$$
\mathcal{M} g=\mathcal{M} \bar{g}
$$

where $\bar{g}$ is the zero extension of $g$ in $\mathbb{R}^{n}$. This maximal function holds the socalled weak $(1,1)$ inequality. More specifically, there exists a positive constant $c=c(n)$ such that

$$
\left|\left\{x \in \mathbb{R}^{n}:(\mathcal{M} g)(x)>\lambda\right\}\right| \leq \frac{c}{\lambda} \int_{\mathbb{R}^{n}}|g(x)| d x
$$

for any $\lambda>0$. As the well-known Muchenhoupt characterization of the $A_{p^{-}}$ weight, the Hardy-Littlewood maximal operator is bounded from weighted Lebesgue space $L_{w}^{p}\left(\mathbb{R}^{n}\right)$ to itself and the $A_{i(\Phi)}$-weight can be classified as follows: Given a Young function $\Phi \in \Delta_{2} \cap \nabla_{2}$, the weight $w$ belongs to the $A_{i(\Phi)}$ class if and only if there exists $c=c(n, \Phi, w)$ such that

$$
\int_{\mathbb{R}^{n}} \Phi(\mathcal{M} g(x)) w(x) d x \leq c \int_{\mathbb{R}^{n}} \Phi(|g(x)|) w(x) d x
$$


for all $g \in L_{w}^{\Phi}\left(\mathbb{R}^{n}\right)$ with compact support in $\mathbb{R}^{n}$. We refer to $[16,17]$ and the references given therein.

The following measure theory in the weighted Orlicz space is needed:

Lemma 2.2. Given a Young function $\Phi \in \Delta_{2} \cap \nabla_{2}$, let $w \in A_{i(\Phi)}$. Assume that $g$ is a nonnegative and measurable function defined on a bounded domain $\Omega$ in $\mathbb{R}^{n}$. Let $\theta>0$ and $\lambda>1$ be constants. Then

$$
g \in L_{w}^{\Phi}(\Omega) \quad \Longleftrightarrow \quad S=\sum_{k \geq 1} \Phi\left(\lambda^{k}\right) w\left(\left\{x \in \Omega: g(x)>\theta \lambda^{k}\right\}\right)<\infty
$$

and

$$
\frac{1}{c} S \leq \int_{\Omega} \Phi(g(x)) w(x) d x \leq c(w(\Omega)+S),
$$

the positive constant $c$ depending only on $\theta, \lambda, \Phi$, and $w$.

The following version of the Calderon-Zygmund-Krylov-Safonov-type covering lemma is used to prove the main theorem. The following lemma can be found in [5, Lemma 5.4] or [23, Lemma 3.4] with slight modifications.

Lemma 2.3. Given a Young function $\Phi \in \Delta_{2} \cap \nabla_{2}$, let $w \in A_{i(\Phi)}$. Let $\Omega$ be a bounded $(\delta, 1)$-Reifenberg flat domain for some small $\delta>0$ and let $C$ and $D$ be measurable sets with $C \subset D \subset \Omega$. Suppose that there exists small $\epsilon>0$ such that

(1) for any $y \in \Omega, w\left(C \cap B_{1}(y)\right)<\epsilon w\left(B_{1}(y)\right)$,

(2) for each $y \in \Omega$ and $r \in(0,1)$,

$$
\text { if } w\left(C \cap B_{r}(y)\right) \geq \epsilon w\left(B_{r}(y)\right), \quad \text { then } B_{r}(y) \cap \Omega \subset D .
$$

Then

$$
w(C) \leq c_{4} \epsilon w(D),
$$

the constant $c_{4}$ depending only on $n, \Phi, w$, and the constant $\frac{1}{1-\delta}$.

\section{Global $W^{1, p}$ estimates}

In this section, we will complete the proof of Theorem 1.4. The following lemma is based on the same method as in the proof in [20, Theorem 4.10].

Lemma 3.1. Let $u \in H_{0}^{1}(\Omega)$ be the weak solution of (1.1). Then there exists a constant $N=N\left(c_{0}, c_{1}, n\right)>1$ such that for each $0<\epsilon<1$ fixed, one can select small $\delta=\delta\left(\epsilon, c_{0}, c_{1}, n, \Phi, w\right) \in\left(0, \frac{1}{8}\right)$ such that if $\mathbf{a}$ is $(\delta, 1)$-vanishing, $\Omega$ is $(\delta, 1)$-Reifenberg flat, and if for $0<r<1$ and $y \in \Omega, B_{r}(y)$ satisfies

$$
w\left(\left\{x \in \Omega: \mathcal{M}\left(|D u|^{2}\right)>N^{2}\right\} \cap B_{r}(y)\right) \geq \epsilon w\left(B_{r}(y)\right),
$$

then we have

$$
B_{r}(y) \cap \Omega \subset\left\{x \in \Omega: \mathcal{M}\left(|D u|^{2}\right)>1\right\} \cup\left\{x \in \Omega: \mathcal{M}\left(|F|^{2}\right)>\delta^{2}\right\} .
$$


We remark that there are similar technical lemmas in the unweighted case for higher order linear problems, see $[7,11,13]$.

From now on, for simplicity and clearance the symbol $c$ denotes a constant that can be explicitly calculated in terms of known quantities. This constant may vary in different occurrences.

Now, we are ready to prove the main theorem.

Proof of Theorem 1.4. Thanks to Lemma 2.1, it suffices to prove that

$$
\left\||D u|^{2}\right\|_{L_{w}^{\Phi}(\Omega)} \leq c, \quad \text { under the assumption }\left\||F|^{2}\right\|_{L_{w}^{\Phi}(\Omega)} \leq \delta^{2} .
$$

In fact, taking

$$
u_{1}=\frac{\delta u}{\sqrt{\left\||F|^{2}\right\|_{L_{w}^{\Phi}(\Omega)}+\sigma}} \quad \text { and } \quad F_{1}=\frac{\delta F}{\sqrt{\left\||F|^{2}\right\|_{L_{w}^{\Phi}(\Omega)}+\sigma}}
$$

in place of $u$ and $F$, respectively, in the problem (1.1), it follows from (1.11) and (1.9) that

$$
\left\|\left|F_{1}\right|^{2}\right\|_{L_{w}^{\Phi}(\Omega)} \leq \delta^{2} \quad \text { and } \quad \int_{\Omega}\left|F_{1}(x)\right|^{2} d x \leq c \delta^{2 \tau_{2}}
$$

where $\tau_{2}=\frac{q_{0}}{q_{1}}$. Therefore if (3.1) is obtained with $D u_{1}$ instead of $D u$, then the proof is completed after letting $\sigma \rightarrow 0$. However, in view of (1.9) and (2.2),

$$
\left\||D u|^{2}\right\|_{L_{w}^{\Phi}(\Omega)}^{\alpha} \leq c \int_{\Omega} \Phi\left(|D u|^{2}\right) w(x) d x \leq c \int_{\Omega} \Phi\left(\mathcal{M}\left(|D u|^{2}\right)\right) w(x) d x
$$

for some $\alpha>0$. Consequently, it suffices to show that, by Lemma 2.2,

$$
S=\sum_{k \geq 1} \Phi\left(N^{2 k}\right) w\left(\left\{x \in \Omega: \mathcal{M}\left(|D u|^{2}\right)>N^{2 k}\right\}\right)<\infty .
$$

We now turn to derive the power decay estimates of the weighted measure of the upper-level set $\left\{x \in \Omega: \mathcal{M}\left(|D u|^{2}\right)>N^{2 k}\right\}$ for $k=1,2,3, \ldots$. To apply Lemma 2.3, first fix $\epsilon$ and take $\delta$ and $N$ as given in Lemma 3.1. Then define the sets

$$
\left\{\begin{array}{l}
C=\left\{x \in \Omega: \mathcal{M}\left(|D u|^{2}\right)>N^{2}\right\} \\
D=\left\{x \in \Omega: \mathcal{M}\left(|D u|^{2}\right)>1\right\} \cup\left\{x \in \Omega: \mathcal{M}\left(|F|^{2}\right)>\delta^{2}\right\} .
\end{array}\right.
$$

Next check its hypotheses. Clearly, $C \subset D \subset \Omega$, and for each $y \in \Omega$,

$$
\begin{aligned}
\frac{w\left(C \cap B_{1}(y)\right)}{w\left(B_{1}(y)\right)} & \stackrel{(1.6)}{\leq} c_{2}\left(\frac{\left|C \cap B_{1}(y)\right|}{\left|B_{1}(y)\right|}\right)^{\tau_{1}} \leq c|C|^{\tau_{1}} \\
& \stackrel{(2.1)}{\leq} c\left(\int_{\Omega}|D u|^{2} d x\right)^{\tau_{1}} \leq c\left(\int_{\Omega}|F|^{2} d x\right)^{\tau_{1}} \stackrel{(3.2)}{\leq} c \delta^{2 \tau_{1} \tau_{2}}<\epsilon
\end{aligned}
$$


for $\delta$ small enough. Because the second condition of Lemma 2.3 is already checked in Lemma 3.1,

$$
\begin{aligned}
& w\left(\left\{x \in \Omega: \mathcal{M}\left(|D u|^{2}\right)>N^{2 k}\right\}\right) \\
\leq & c_{4} \epsilon w\left(\left\{x \in \Omega: \mathcal{M}\left(|D u|^{2}\right)>1\right\}\right) \\
& +c_{4} \epsilon w\left(\left\{x \in \Omega: \mathcal{M}\left(|F|^{2}\right)>\delta^{2} N^{2(k-i)}\right\}\right) .
\end{aligned}
$$

On the other hand, the main problem (1.1) has the invariance property from normalization (see Lemma 2.1) and therefore the same result (3.3) may be obtained for $\left(\frac{u}{N}, \frac{F}{N}\right),\left(\frac{u}{N^{2}}, \frac{F}{N^{2}}\right),\left(\frac{u}{N^{3}}, \frac{F}{N^{3}}\right), \ldots$, inductively. From this iteration argument (for further details see, [20, Corollary 4.11]), the following power decay estimates are obtained:

$$
\begin{aligned}
& w\left(\left\{x \in \Omega: \mathcal{M}\left(|D u|^{2}\right)>N^{2 k}\right\}\right) \\
\leq & \epsilon_{1}^{k} w\left(\left\{x \in \Omega: \mathcal{M}\left(|D u|^{2}\right)>1\right\}\right) \\
& +\sum_{i=1}^{k} \epsilon_{1}^{i} w\left(\left\{x \in \Omega: \mathcal{M}\left(|F|^{2}\right)>\delta^{2} N^{2(k-i)}\right\}\right)
\end{aligned}
$$

for $k=1,2, \ldots$, where $\epsilon_{1}=c_{4} \epsilon$. Then a direct computation yields

$$
\begin{aligned}
S= & \sum_{k \geq 1} \Phi\left(N^{2 k}\right) w\left(\left\{x \in \Omega: \mathcal{M}\left(|D u|^{2}\right)>N^{2 k}\right\}\right) \\
\leq & \sum_{k \geq 1} \Phi\left(N^{2 k}\right) \epsilon_{1}^{k} w\left(\left\{x \in \Omega: \mathcal{M}\left(|D u|^{2}\right)>1\right\}\right) \\
& \quad+\sum_{k \geq 1} \Phi\left(N^{2 k}\right) \sum_{i=1}^{k} \epsilon_{1}^{i} w\left(\left\{x \in \Omega: \mathcal{M}\left(|F|^{2}\right)>\delta^{2} N^{2(k-i)}\right\}\right) \\
= & : S_{1}+S_{2} .
\end{aligned}
$$

Recall the following property of $\Phi \in \Delta_{2}$ : There exists a constant $\mu_{1}$, depending only on $\Phi$ and $N$ such that $\Phi\left(N^{2}\right) \leq \mu_{1} \Phi(1)$, and therefore

$$
\Phi\left(N^{2 k}\right) \leq \mu_{1}^{k} \Phi(1), \quad k=1,2,3, \ldots
$$

$S_{1}$ is estimated as follows:

$$
S_{1} \leq \sum_{k \geq 1}\left(\Phi(1) \mu_{1}^{k} \epsilon_{1}^{k} w(\Omega)\right) \leq c \sum_{k \geq 1}\left(\mu_{1} \epsilon_{1}\right)^{k} .
$$

On the other hand,

$$
\begin{aligned}
S_{2} & =\sum_{k \geq 1} \Phi\left(N^{2(k-i)} N^{2 i}\right) \sum_{i=1}^{k} \epsilon_{1}^{i} w\left(\left\{x \in \Omega: \mathcal{M}\left(|F|^{2}\right)>\delta^{2} N^{2(k-i)}\right\}\right) \\
& \leq \sum_{i \geq 1} \sum_{k \geq i} \Phi\left(N^{2(k-i)}\right) \mu_{1}^{i} \epsilon_{1}^{i} w\left(\left\{x \in \Omega: \mathcal{M}\left(|F|^{2}\right)>\delta^{2} N^{2(k-i)}\right\}\right)
\end{aligned}
$$




$$
\begin{aligned}
& \leq c \sum_{i \geq 1}\left(\mu_{1} \epsilon_{1}\right)^{i} \sum_{k \geq i} \Phi\left(N^{2(k-i)}\right) w\left(\left\{x \in \Omega: \mathcal{M}\left(|F|^{2}\right)>\delta^{2} N^{2(k-i)}\right\}\right) \\
& \leq c \sum_{i \geq 1}\left(\mu_{1} \epsilon_{1}\right)^{i} \sum_{j \geq 0} \Phi\left(N^{2 j}\right) w\left(\left\{x \in \Omega: \mathcal{M}\left(\left|\frac{F}{\delta}\right|^{2}\right)>N^{2 j}\right\}\right) \\
& \stackrel{(2.3)}{\leq} c \sum_{i \geq 1}\left(\mu_{1} \epsilon_{1}\right)^{i} \int_{\Omega} \Phi\left(\mathcal{M}\left(\left|\frac{F}{\delta}\right|^{2}\right)\right) w(x) d x \\
& \stackrel{(2.2),(1.9)}{\leq} c \sum_{i \geq 1}\left(\mu_{1} \epsilon_{1}\right)^{i}\left\|\frac{|F|^{2}}{\delta^{2}}\right\|_{L_{w}^{\Phi}(\Omega)}^{\stackrel{(3.1)}{\leq} c \sum_{i \geq 1}\left(\mu_{1} \epsilon_{1}\right)^{i} .}
\end{aligned}
$$

Therefore,

$$
S \leq c \sum_{k \geq 1}\left(\mu_{1} \epsilon_{1}\right)^{k}
$$

where $\epsilon_{1}=c_{4} \epsilon$, as in Lemma 2.3.

First take sufficiently small $\epsilon>0$ to get

$$
\mu_{1} \epsilon_{1}<1
$$

Then one can select correspondingly small $\delta=\delta\left(c_{0}, c_{1}, n, \Phi, w\right)>0$ from Lemma 3.1. This completes the proof.

\section{References}

[1] E. Acerbi and G. Mingione, Gradient estimates for a class of parabolic systems, Duke Math. J. 136 (2007), no. 2, 285-320.

[2] P. Baroni, Lorentz estimates for degenerate and singular evolutionary systems, J. Differential Equations 255 (2013), no. 9, 2927-2951.

[3] P. Baroni, A. Di Castro, and G. Palatucci, Global estimates for nonlinear parabolic equations, J. Evol. Equ. 13 (2013), no. 1, 163-195.

[4] S. Byun, J. Ok, and S. Ryu, Global gradient estimates for general nonlinear parabolic equations in nonsmooth domains, J. Differential Equations 254 (2013), no. 11, 42904326.

[5] S. Byun and D. K. Palagachev, Weighted $L^{p}$-estimates for elliptic equations with measurable coefficients in nonsmooth domains, Potential Anal. 41 (2014), no. 1, 51-79.

[6] S. Byun, D. K. Palagachev, and S. Ryu, Weighted $W^{1, p}$ estimates for solutions of non-linear parabolic equations over non-smooth domains, Bull. London Math. Soc. 45 (2013), no. 4, 765-778.

[7] S. Byun and S. Ryu, Gradient estimates for higher order elliptic equations on nonsmooth domains, J. Differential Equations 250 (2011), no. 1, 243-263.

[8] Global weighted estimates for the gradient of solutions to nonlinear elliptic equations, Ann. Inst. H. Poincaré Anal. Non Linéaire 30 (2013), no. 2, 291-313.

[9] S. Byun and L. Wang, Elliptic equations with BMO nonlinearity in Reifenberg domains, Adv. Math. 219 (2008), no. 6, 1937-1971.

[10] L. A. Caffarelli and I. Peral, On $W^{1, p}$ estimates for elliptic equations in divergence form, Comm. Pure Appl. Math. 51 (1998), no. 1, 1-21.

[11] H. Dong and D. Kim, Higher order elliptic and parabolic systems with variably partially BMO coefficients in regular and irregular domains, J. Funct. Anal. 261 (2011), no. 11, 3279-3327. 
$[12] \_, L_{p}$ solvability of divergence type parabolic and elliptic systems with partially BMO coefficients, Calc. Var. Partial Differential Equations 40 (2011), no. 3-4, 357-389.

[13] _ The conormal derivative problem for higher order elliptic systems with irregular coefficients, Recent advances in harmonic analysis and partial differential equations, 6997, Contemp. Math., 581, Amer. Math. Soc., Providence, RI, 2012.

[14] F. Duzaar, G. Mingione, and K. Steffen, Parabolic systems with polynomial growth and regularity, Mem. Amer. Math. Soc. 214 (2011), no. 1005, x+118 pp.

[15] G. Di Fazio, $L^{p}$ estimates for divergence form elliptic equations with discontinuous coefficients, Boll. Un. Mat. Ital A (7) 10 (1996), no. 2, 409-420.

[16] A. Fiorenza and M. Krbec, Indices of Orlicz spaces and some applications, Comment. Math. Univ. Carolin. 38 (1997), no. 3, 433-451.

[17] V. Kokilashvili and M. Krbec, Weighted Inequalities in Lorentz and Orlicz Spaces, World Scientific Publishing Co., Inc., River Edge, NJ, 1991

[18] N. V. Krylov, Parabolic and elliptic equations with VMO coefficients, Comm. Partial Differential Equations 32 (2007), no. 1-3, 453-475

[19] Lectures on elliptic and parabolic equations in Sobolev spaces, Graduate Studies in Mathematics, 96. American Mathematical Society, Providence, RI, 2008. xviii+357 pp.

[20] T. Mengesha and N. C. Phuc, Weighted and regularity estimates for nonlinear equations on Reifenberg flat domains, J. Differential Equations 250 (2011), no. 5, 2485-2507.

[21] _ Global Estimates for quasilinear elliptic equations on Reifenberg flat domains, Arch. Ration. Mech. Anal. 203 (2012), no. 1, 189-216.

[22] D. K. Palagachev and L. G. Softova, A priori estimates and precise regularity for parabolic systems with discontinuous data, Discrete Contin. Dyn. Syst. 13 (2005), no. 3, 721-742.

[23] N. C. Phuc, Nonlinear Muckenhoupt-Wheeden type bounds on Reifenberg flat domains, with applications to quasilinear Riccati type equations, Adv. Math. 250 (2014), 387-419.

[24] L. G. Softova, Morrey-type regularity of solutions to parabolic problems with discontinuous data, Manuscripta Math. 136 (2011), no. 3-4, 365-382.

[25] T. Toro, Doubling and flatness: geometry of measures, Notices Amer. Math. Soc. 44 (1997), no. 9, 1087-1094.

[26] L. Wang and F. Yao, Global regularity for higher order divergence elliptic and parabolic equations, J. Funct. Anal. 266 (2014), no. 2, 792-813.

[27] L. Wang, F. Yao, S. Zhou, and H. Jia, Optimal regularity theory for the Poisson equation, Proc. Amer. Math. Soc. 137 (2009), no. 6, 2037-2047.

Department of Mathematics

University of SeOUl

SEOUl 130-743, Korea

E-mail address: seungjinryu@uos.ac.kr 